\title{
Once-weekly Subcutaneous Administration of Bortezomib in Patients with Multiple Myeloma
}

\author{
Liang Wang ${ }^{\star}$, Ke-Feng Wang ${ }^{\&}$, Bo-Yang Chang ${ }^{\star}$, Xiao-Qin Chen, Zhong-Jun \\ Xia*
}

\begin{abstract}
In patients with multiple myeloma (MM), once-weekly intravenous injection or twice-weekly subcutaneous injection (SC) of bortezomib has been proven to offer non-inferior efficacy to standard twice-weekly intravenous administration, with an improved safety profile. However, whether once-weekly $\mathrm{SC}$ bortezomib can further reduce the incidence rate of peripheral neuropathy $(\mathrm{PN})$ and not compromise the efficacy remains to be investigated. 25 patients of MM treated with once-weekly SC bortezomib were reviewed in this study. The median treatment cycles were 4 (range, $2-9$ cycles). Complete response (CR) rate was 52\%, $\geq$ very good partial response (VGPR) rate was $72 \%$, and $\geq$ partial response (PR) rate was $84 \%$. 1-year and 2-year PFS rate was $63.0 \%$ and 34.3\%, respectively, and 2-year OS rate was $100 \%$. Any grade of $\mathrm{PN}$ was reported in 9 patients $(36.0 \%)$, with 7 patients $(28.0 \%)$ had grade $1 \mathrm{PN}$, and 2 patients $(\mathbf{8 . 0 \%})$ had grade $2 \mathrm{PN}$. No patients reported grade $3 / 4 \mathrm{PN}$ in this cohort. In conclusion, once-weekly subcutaneous administration of bortezomib offers excellent efficacy with a further improved safety profile, especially with regard to PN. It needs to be validated in future prospective randomized trials.
\end{abstract}

Keywords: Bortezomib - subcutaneous - multiple myeloma - peripheral neuropathy - efficacy

Asian Pac J Cancer Prev, 16 (5), 2093-2098

\section{Introduction}

Multiple myeloma (MM) is a fatal plasma cell disease that accounts for $1 \%$ of all cancers and $10 \%$ of hematological malignancies. It primarily affects older individuals, and the median age at diagnosis is 70 years and nearly two-thirds of MM patients are more than 65 years of age when they are first diagnosed (Sonneveld et al., 2013). The outcome of MM has significantly improved in the last decade because myeloma treatment is developing rapidly (Kumar et al., 2008). The introduction of the proteasome inhibitor bortezomib and the immunomodulatory drugs such as thalidomide and lenalidomide have contributed to improvements in overall survival in patients with multiple myeloma (Raab et al., 2009; Wang et al., 2012). Bortezomib-based therapies are suggested as standards of care in patients with newly diagnosed and relapsed multiple myeloma (Engelhardt et al., 2010).

The first recommended dose and schedule of bortezomib is $1.3 \mathrm{mg} / \mathrm{m}^{2}$ administered as a bolus intravenous injection (IV) on days 1,4,8, and 11 of 21-day cycles. This regimen is active and well tolerated. However, one of its most frequent and potentially disabling sideeffects is the development of a painful, sensory peripheral neuropathy (PN) (Richardson et al., 2006; Richardson et al., 2012; Voorhees et al., 2013) often requiring dose modification or discontinuation of bortezomib, which negatively affects clinical endpoints and quality of life (Mateos, 2010). Grade 1 and 2 bortezomib-induced PN can occur in 27-75\% of patients with recurrent MM and in 25$33 \%$ of those with newly diagnosed MM, whereas grade 3 and 4 PN might affect $0-30 \%$ of patients with recurrent disease and $0-18 \%$ of those with newly diagnosed disease (Badros et al., 2007). Subsequent trials evaluated the efficacy and safety of once-weekly IV bortezomib in patients with MM (Bringhen et al., 2010; Reeder et al., 2010; Koh et al., 2014), and concluded that once-weekly administration of bortezomib can significantly reduce the incidence rate of $\mathrm{PN}$, and did not appear to influence the efficacy.

As an alternative to intravenous delivery, subcutaneous administration (SC) of bortezomib could be a good option for patients, particularly those with poor venous access. A randomized phase 3 controlled trial (Moreau et al., 2011) comparing the efficacy and safety of twice-weekly SC versus IV of bortezomib in patients with relapsed $\mathrm{MM}$ and found that $\mathrm{PN}$ of any grade (56 [38\%] vs 39 [53\%]; $p=0.044)$, grade 2 or worse (35 [24\%] vs 30

Department of Hematologic Oncology, Sun Yat-sen University Cancer Center, State Key Laboratory of Oncology in South China, Collaborative Innovation Center for Cancer Medicine, Guangzhou, Guangdong, People’s Republic of China ${ }^{\star} E q u a l$ contributors *For correspondence: xiazhj@21cn.com 
Liang Wang et al

[41\%]; $p=0.012$ ), and grade 3 or worse (nine [6\%] vs 12 [16\%]; $p=0.026$ ) was significantly less common with SC than with IV. SC was locally well tolerated and did not compromise the efficacy, leading to the FDA approval of $\mathrm{SC}$ bortezomib in patients with MM.

However, whether once-weekly SC bortezomib can further reduce the incidence rate of $\mathrm{PN}$ and not compromise the efficacy remains to be investigated. This strategy of bortezomib administration has been used since 2012 in our center, and herein, we reported the efficacy and safety results of a cohort of 25 patients with MM who received once-weekly SC bortezomib.

\section{Materials and Methods}

\section{Patients}

From January 2012 to July 2014, 25 patients with newly diagnosed MM or relapsed/refractory MM (patients were not exposed to prior bortezomib) treated with once-weekly SC bortezomib were included in this study. Patients with PN of any grade before the first dose of bortezomib were excluded from this study. Informed consent for the collection of medical information was obtained from all patients at their first visit, and this study was approved by Sun Yat-sen University Cancer Center Research Ethics Board.

\section{Treatments}

All patients were treated with VD (bortezomib 1.3mg/ $\mathrm{m}^{2}$, SC, once-weekly, and dexamethasone $40 \mathrm{mg}$, onceweekly, repeated every 4 weeks), VTD (thalidomide $100 \mathrm{mg} /$ day for 4 weeks, the dosage and schedule of VD were the same as above, repeated every 4 weeks), or CyBorD (cyclophosphamide, $300 \mathrm{mg} / \mathrm{m}^{2}$, po, once-weekly, the dosage and schedule of VD were the same as above, repeated every 4 weeks). For those transplant-eligible patients, high dose melphalan and autologous stem cell transplantation (HDT-ASCT) were recommended after four cycles of treatment. For transplant-ineligible patients, at least four to six cycles of induction therapy were given before initiation of maintenance therapy. If minimal response was not attained after at least 2 cycles of treatment, second-line regimens such as VRD (bortezomib $1.3 \mathrm{mg} / \mathrm{m}^{2}, \mathrm{SC}$, once-weekly, lenalidomide $25 \mathrm{mg} \mathrm{d} 1-21$, and dexamethasone $40 \mathrm{mg}$, once-weekly, repeated every 4 weeks) or DVD (pegylated liposomal doxorubicin $25 \mathrm{mg} / \mathrm{m}^{2}, \mathrm{~d} 1$, bortezomib $1.3 \mathrm{mg} / \mathrm{m}^{2}, \mathrm{SC}$, once-weekly, and dexamethasone $40 \mathrm{mg}$, once-weekly, repeated every 4 weeks) were used. Thromboprophylaxis (aspirin 100mg/d) was given for all patients treated with thalidomide or lenalidomide. Subcutaneous bortezomib injections were administered at $2.5 \mathrm{mg} / \mathrm{mL}(3.5 \mathrm{mg}$ bortezomib reconstituted with $1.4 \mathrm{~mL}$ normal [0.9\%] saline) to limit the volume injected. Subcutaneous injection sites were the thighs or abdomen; sites were rotated for successive injections. Injections at the same site within a cycle were avoided. Alternation between right and left abdomen, upper and lower quadrant, or right and left thigh, proximal and distal sites, was recommended.

\section{Efficacy evaluation}

We assessed the response to treatment using criteria of the International Myeloma Working Group Uniform Response Criteria (IMWG) (Durie et al., 2006). For all patients, we collected blood and 24-h urine samples for M-protein measurement at baseline, every 4 weeks (day 1 of each cycle) during the treatment period, at the endof-treatment visit, and then every 8 weeks until disease progression. In patients with $100 \%$ M-protein reduction by electrophoresis, determination of $\mathrm{CR}$ required

\section{Table 1. Clinical Characteristics of 25 Patients with Multiple Myeloma}

\begin{tabular}{|c|c|c|}
\hline Parameters & & Patients $(\mathrm{N}=25)(\%)$ \\
\hline \multirow[t]{2}{*}{ Age } & $\geq 65$ & $9(36.0)$ \\
\hline & $<65$ & $16(64.0)$ \\
\hline \multirow[t]{2}{*}{ Gender } & Male & $19(76.0)$ \\
\hline & Female & $6(24.0)$ \\
\hline \multirow[t]{2}{*}{ Disease status } & De novo & $17(68.0)$ \\
\hline & Refractory/relapsed & $8(32.0)$ \\
\hline \multirow[t]{3}{*}{ ISS stage } & I & $5(20.0)$ \\
\hline & II & $6(24.0)$ \\
\hline & III & $14(56.0)$ \\
\hline \multirow[t]{2}{*}{ Renal dysfunction } & Yes & $4(16.0)$ \\
\hline & No & $21(84.0)$ \\
\hline \multirow[t]{2}{*}{ Immunoparesis } & Yes & $18(72.0)$ \\
\hline & No & $7(28.0)$ \\
\hline \multirow[t]{4}{*}{ M protein type } & $\operatorname{IgG}$ & $13(52.0)$ \\
\hline & $\operatorname{IgA}$ & $5(20.0)$ \\
\hline & Light chain & $5(20.0)$ \\
\hline & Non-secretory & $2(8.0)$ \\
\hline \multirow[t]{5}{*}{ Best response to once-weekly SC bortezomib } & $\mathrm{CR}$ & $13(52.0)$ \\
\hline & VGPR & $5(20.0)$ \\
\hline & PR & $3(12.0)$ \\
\hline & SD & $3(12.0)$ \\
\hline & PD & $1(4.0)$ \\
\hline
\end{tabular}

*Abbreviations: ISS, international staging system; SC, subcutaneously; CR, complete response; VGPR, very good partial response; PR, partial response; $\mathrm{SD}$, stable disease; $\mathrm{PD}$, progressive disease 
immunofixation and bone marrow examination. Bone marrow aspiration and biopsy were done for all patients at baseline and as needed for confirmation of CR or diagnosis of progression. Adverse events were graded according to National Cancer Institute common terminology criteria for adverse events (version 3.0).

\section{Statistical analysis}

Progression free survival (PFS) was calculated from the date of first dose bortezomib to the date of disease progression or death and was censored at the date of the last follow-up visit. Overall survival (OS) was calculated from the date of diagnosis to the date of death from any cause and was censored at the date of the last follow-up visit. All statistical analysis was performed using PASW Statistics 18.0 software (Apache Software Foundation, Forest Hill, Md). Survival analysis was performed using the Kaplan-Meier method and log-rank test. $p<0.05$ was considered statistically significant.

\section{Results}

\section{Patients}

The patients' characteristics were shown in Table 1. The median age at diagnosis was 61 years old (range, $44-73$ ), and $64 \%$ patients were younger than 65 years

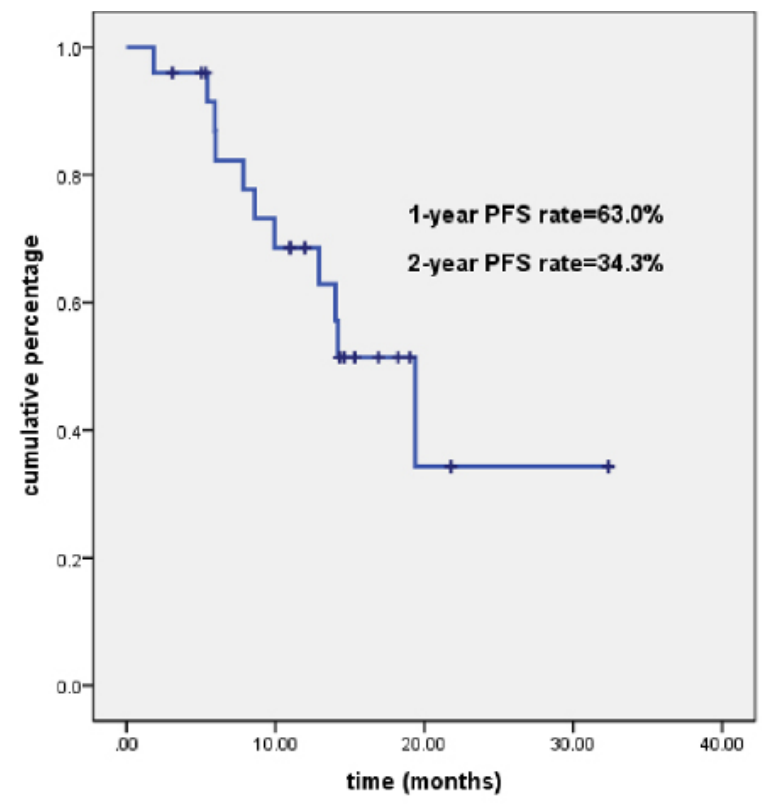

Figure 1. Progression-free Survival Curve for the whole Cohort of Patients with MM. This PFS time was calculated from the initiation of first dose bortezomib treatment old. In this cohort, $8(32 \%)$ patients were previously treated with non-bortezomib-based chemotherapy (mainly VAD (vincristine, adriamycin, and dexamethasone), DVD (pegylated liposomal doxorubicin, vincristine, and dexamethasone) or MPT (melphalan, prednisone, and thalidomide)), among whom 4 patients had progressive disease, 2 patients had stable disease, and the other 2 patients had relapsed disease.According to the international staging system (ISS), 56\% patients had stage III disease, and $16 \%$ patients had renal dysfunction. Most patients in this cohort (72\%) had immunoparesis. The predominant type of M protein was $\operatorname{IgG}(52 \%)$, followed by $\operatorname{IgA}(20 \%)$ and light chain $(20 \%)$.

\section{Treatments and efficacy}

All patients in this cohort received once-weekly SC bortezomib $\left(1.3 \mathrm{mg} / \mathrm{m}^{2}\right)$ based regimens, namely VDT (20 patients), VD (4 patients), and CyBorD (1 patient). The median treatment cycles were 4 (range, 2-9 cycles). As is demonstrated in Table 1, for the whole cohort, CR rate was $52 \%, \geq \mathrm{VGPR}$ rate was $72 \%$, and $\geq \mathrm{PR}$ rate was $84 \%$. There were no significant differences between patients with de novo disease and patients with refractory/relapsed disease in CR rate $(52.9 \%$ vs. $50 \%, p>0.05)$, $\geq$ VGPR rate $(76.5 \%$ vs. $62.5 \%, p>0.05)$, or $\geq \mathrm{PR}$ rate $(82.3 \% v s$. $87.5 \%, p>0.05)$.

At a median follow up time of 15.0 months after initiation of bortezomib treatment (range, 3.1-32.4 months), 11 patients had disease progression, and the 1 -year and 2-year PFS rate was $63.0 \%$ and $34.3 \%$, respectively. Among those 11 patients, 6 patients were retreated with once-weekly subcutaneous administration of bortezomib $\left(1.3 \mathrm{mg} / \mathrm{m}^{2}\right)$ based regimens, and $33.3 \%$ patients got PR, $50.0 \%$ patients had stable disease (SD), and one patient had progressive disease. In our cohort, only four patients received HDT-ASCT ( 2 with de novo disease and 2 with relapsed disease), and after HDT-ASCT, the $\mathrm{CR}$ rate was $50 \%, \geq \mathrm{VGPR}$ rate was $75 \%$, and $\geq \mathrm{PR}$ rate was $100 \%$. Of those four patients, three patients $(75 \%)$ had disease progression at 10.0, 11.0, and 14.2 months after HDT-ASCT, respectively.

As was depicted in Figure 1, the best response status after bortezomib-based treatment (CR vs. VGPR+PR vs. $\mathrm{SD}+\mathrm{PD})$ significantly correlated with PFS $(p=0.026)$, but the disease status (de novo vs. refractory/relapsed) did not correlated with PFS $(p=0.903)$. At a median follow up time of 18.7 months after the diagnosis of MM (range, 5.3-110.0 months), all patients were alive, and 2-year OS rate was $100 \%$.

Table 2. Adverse Events Related to Subcutaneous Administration of Bortezomib

\begin{tabular}{lcccc}
\hline Adverse events & \multicolumn{3}{c}{ Patients (N=25) (\%) } \\
\cline { 2 - 5 } & Grade 1 & Grade 2 & $\geq$ grade 3 & Any grade \\
\hline Peripheral sensory neuropathy & $7(28.0)$ & $2(8.0)$ & 0 & $9(36.0)$ \\
Neuralgia & $2(8.0)$ & 0 & 0 & $2(8.0)$ \\
Diarrhea & $4(16.0)$ & 0 & 0 & $4(16.0)$ \\
Herpes zoster infection & $1(4.0)$ & 0 & 0 & $1(4.0)$ \\
Bortezomib dose reductions because of PN & NA & NA & NA & $1(4.0)$ \\
Treatment discontinuations because of PN & NA & NA & NA & $1(4.0)$ \\
Administration site conditions & $6(24.0)$ & $1(4.0)$ & 0 & $7(28.0)$ \\
\hline
\end{tabular}

*Abbreviations: PN, peripheral neuropathy; NA, not applicable. 

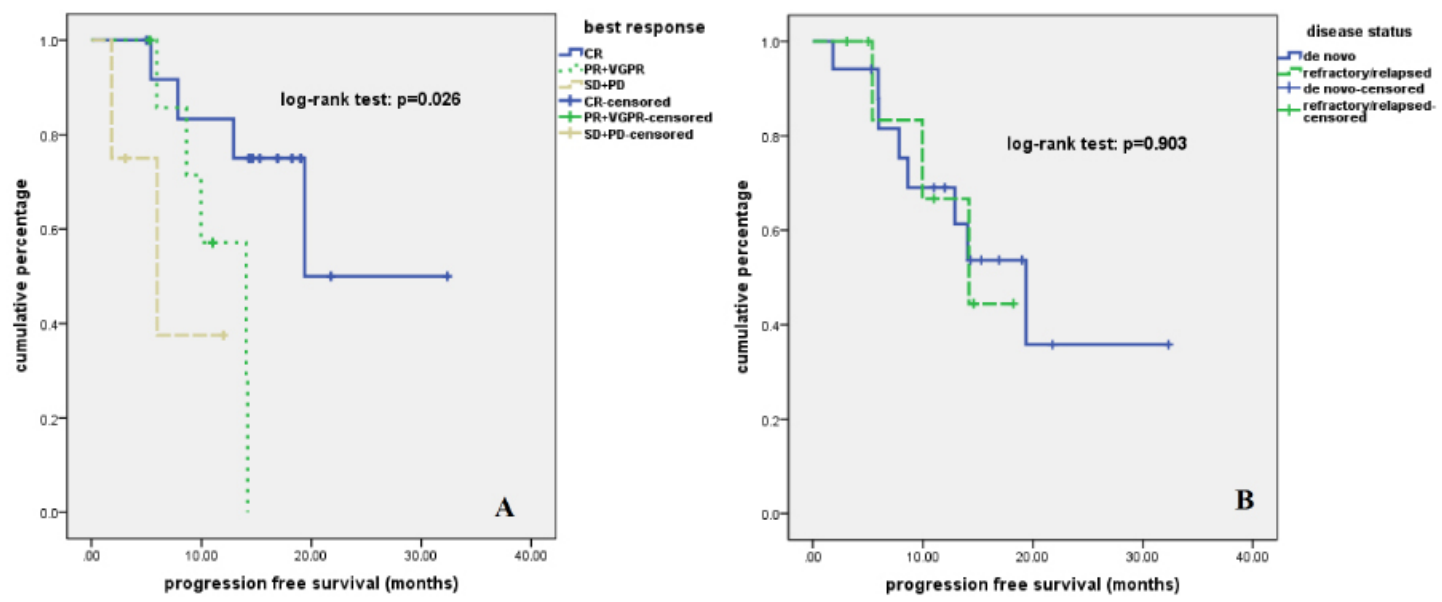

Figure 2. Progression-free Survival Curves for Different Subgroup Patients. This PFS Time was Calculated from the Initiation of First dose Bortezomib Treatment. A, The best response status to bortezomib treatment was significantly correlated with PFS ( $p=0.026$ ); B, The disease status (de novo vs. refractory/relapsed) was not correlated with PFS $(p=0.903$ )

Toxicities related to once-weekly subcutaneous administration of bortezomib

Table 2 listed the most common adverse events that were considered to be related with SC bortezomib treatment. Any grade of peripheral sensory neuropathy (PN) was reported in 9 patients $(36.0 \%)$, with 7 patients (28.0\%) had grade $1 \mathrm{PN}$, and 2 patients $(8.0 \%)$ had grade $2 \mathrm{PN}$. No patients reported grade $3 / 4 \mathrm{PN}$ in this cohort. 2 patients $(8.0 \%)$ had mild neuralgia (grade 1$)$. At the time of data cutoff, $55.6 \%$ of peripheral-neuropathy events had either resolved $(44.4 \%)$ or decreased by at least one toxicity grade $(11.1 \%)$ within a median of 3 months. Four patients (16.0\%) had mild diarrhea, which resolved after supportive treatment. One patient developed herpes zoster infection after 6 cycles of VTD, and symptoms improved significantly after treatment with antiviral treatment. In this cohort, bortezomib was discontinued in one patient due to grade $2 \mathrm{PN}$ with neuralgia, and reduced dose of bortezomib $\left(1.0 \mathrm{mg} / \mathrm{m}^{2}\right)$ was given in one patient due to grade 2 PN. 7 patients $(28.0 \%)$ had one or more subcutaneous injection-site reactions, and the most common reaction was mild redness (grade 1 in $24.0 \%$ patients). 1 patient had grade 2 injection-site reaction (painful subcutaneous mass). All reactions resolved completely in a median of 10 days (range 2-48).

\section{Discussion}

Previous studies have confirmed the excellent efficacy of bortezomib in the treatment of multiple myeloma both in newly diagnosed patients and relapsed/refractory patients (Raab et al., 2009; Reeder et al., 2010; Bringhen et al., 2010; Engelhardt et al., 2010; Sonneveld et al., 2012; Nooka et al., 2013; Sonneveld et al., 2013). When given as twice-weekly IV, bortezomib can cause any grade of PN in more than half patients (Richardson et al., 2006; Badros et al., 2007; Mateos, 2010; Koh et al., 2014), usually leading to dosage reduction or treatment discontinuation, which might compromise the efficacy. Several clinical trials have evaluated the benefit of prolonging the administration interval of bortezomib as once-weekly intravenous injection, and concluded that this strategy can significantly reduce the incidence rate of $\mathrm{PN}$, and did not appear to influence the efficacy (Bringhen et al., 2010; Reeder et al., 2010). Similarly, the SC administration of bortezomib as twice-weekly injection was found to be noninferior in efficacy to standard twice-weekly intravenous administration, with an improved safety profile (Moreau et al., 2011). Our report was the first to evaluate the efficacy and safety profile of combining prolonged administration interval and subcutaneous administration (once-weekly SC injection of bortezomib), and it was demonstrated that this strategy may further reduce the incidence rate of PN and improve other safety profile without affecting efficacy.

Multiple myeloma usually affects elderly patients with the median age at first diagnosis reported to be 70 years old in western countries (Raab et al., 2009; Morgan, 2013; Roschewski et al., 2013). However, more and more younger patients are diagnosed as MM in China, with the median age at first diagnosis reported to be 59 years old (Lu et al., 2014), which was consistent with our cohort (median age was 61 years old). For elderly patients with $\mathrm{MM}$, the recommended induction regimens usually were melphalan-based. However, due to lack of this drug in China for nearly three years, patients with MM in our center were treated with VDT or VD as first-line therapy. In our study, more than $80 \%$ of patients treated with VTD got PR or better, but the incidence rate of any grade PN was only $30 \%$ and no patients had grade 3 or higher PN, indicating the strategy using once-weekly administration of bortezomib is efficacious and can further improve the safety profile.

In our study, 8 patients were previously treated with non-bortezomib-based chemotherapy regimens. The efficacy of once-weekly SC bortezomib-based therapy in this subgroup of patients was similar to those with de novo MM, and as is shown in Figure 2B, there was no significant difference in PFS between de novo patients and refractory/relapsed patients, indicating bortezomib can benefit patients with MM during the whole course of treatment, no matter as first-line or salvage therapy. Furthermore, 6 patients who had progressive disease after bortezomib treatment were retreated with once-weekly subcutaneous administration of bortezomib $\left(1.3 \mathrm{mg} / \mathrm{m}^{2}\right)$ 
based regimens, and still one third patients got PR, and half patients had stable disease (SD), with the overall disease control rate of $83.3 \%$. Recently, Knopf KB et al (Knopf et al., 2014) performed a meta-analysis evaluating the efficacy and safety of bortezomib re-treatment in 1051 patients with multiple myeloma, and showed that pooled, weighted average ORR was $39.1 \%$ (95\% confidence interval, 30.8\%-47.4\%), which was consistent with our results, suggesting in an era of new and emerging treatment options for relapsed and/or refractory myeloma, bortezomib retreatment might be a highly effective option in previously treated patients.

Nowdays, studies have found that approximately $25 \%$ of MM patients have cytogenetic abnormalities that are associated with a high risk of disease progression and very poor prognosis. High-risk cytogenetic profiles include del(17p), t(4;14), and/or t (14;16) (Bergsagel et al., 2013). In our study, only 10 patients had available data about the cytogenetic features, among whom 1 patient had both $\operatorname{del}(17 \mathrm{p})$ and $1 \mathrm{q} 21$ amplification, 1 patient had $\mathrm{t}(4 ; 14)$, and the remaining 8 patients had normal cytogenetics. The patient with del(17p) and 1q21 amplification was resistant to bortezomib- or lenalidomide-based treatments ( 2 cycles of VTD, 2 months of Rd), but had stable disease after 4 cycles of VAD, and remained in SD status for 10 months with thalidomide maintenance. The patient with $t(4 ; 14)$ got CR after 2 cycle of VTD, and remained in CR status at the last visit (14 months after bortezomib treatment), indicating bortezomib treatment can overcome the prognostic value of $t(4 ; 14)$. Our findings were consistent with the results of previous study (Avet-Loiseau et al., 2010), in which it was found that bortezomib significantly improves the prognosis (in terms of both EFS and OS) of patients with $\mathrm{t}(4 ; 14)$, compared with patients treated with vincristine, doxorubicin, and dexamethasone induction therapy, but in contrast, no improvement was observed for $\operatorname{del}(17 p)$ patients. Thus, for patients with del(17p), novel agents or regimens should be evaluated in future studies.

Increasing evidences have demonstrated that the depth of response to treatment correlates significantly with prognosis (Chanan-Khan and Giralt, 2010; Harousseau, Attal, and Avet-Loiseau, 2009), thus it is recommended to use HDT-ASCT as first-line treatment in transplanteligible patients in order to improve the depth of response. As is shown in Figure 2A, patients who got CR had significantly better PFS than those who only got PR or VGPR. Thus, the pursuit of highly deep response should be the goal of treatment for MM patients. Recently, more and more studies have evaluate the role of stringent CR (sCR) in MM patients (Kapoor et al., 2013) and found that improved long-term outcome was seen after ASCT with achievement of sCR when compared with lesser degrees of responses, thus suggesting that myeloma trials reporting the response rates should identify patients achieving sCR and CR separately, owing to markedly disparate outcomes of the two categories. However, due to lack of this technique in our center, sFLC was not routinely tested for MM patients. In future clinical trials, we will perform sFLC test to evaluate the accurate response to once-weekly SC bortezomib.

We did not performed pharmacokinetic and pharmacodynamic substudy in this cohort of patients. As was reported in previous study (Moreau et al., 2011), mean maximum plasma concentration ( $\mathrm{Cmax}$ ) was much lower after subcutaneous than intravenous injection, but the mean bortezomib systemic exposure (AUClast) was similar, and also mean percentage inhibition of $20 \mathrm{~S}$ proteasome activity (Emax) and area under the effect-time curve were similar. Thus, we hypothesized that onceweekly SC bortezomib can further decrease the Cmax, which may partially explain the much lower incidence rate of $\mathrm{PN}$ in our cohort, but the AUClast Emax were similar between once-weekly SC injection and twiceweekly intravenous injection, which can be deferred from the excellent efficacy of our strategy, and it needs to be validated in prospective randomized trials.

In conclusion, this retrospective study showed that once-weekly SC administration of bortezomib offered excellent efficacy with a further improved safety profile, especially peripheral neuropathy. It needs to be validated in future prospective randomized trials, and suggests that once-weekly SC administration may be a promising alternative to once-weekly intravenous administration or twice-weekly SC administration.

\section{Acknowledgements}

This work received grant support from Outstanding Young Talents Project of Sun Yat-sen University Cancer Center (No. 04190101\#).

\section{References}

Avet-Loiseau H, Leleu X, Roussel M, et al (2010). Bortezomib plus dexamethasone induction improves outcome of patients with $\mathrm{t}(4 ; 14)$ myeloma but not outcome of patients with $\operatorname{del}(17 \mathrm{p})$. J Clin Oncol, 28, 4630-4.

Badros A, Goloubeva O, Dalal J S, et al (2007). Neurotoxicity of bortezomib therapy in multiple myeloma: a single-center experience and review of the literature. Cancer, 110, 1042-9.

Bergsagel PL, Mateos MV, Gutierrez NC, Rajkumar SV, San Miguel JF (2013). Improving overall survival and overcoming adverse prognosis in the treatment of cytogenetically high-risk multiple myeloma. Blood, 121, 884-92.

Bringhen S, Larocca A, Rossi D, et al (2010). Efficacy and safety of once-weekly bortezomib in multiple myeloma patients. Blood, 116, 4745-53.

Chanan-Khan AA, and Giralt S (2010). Importance of achieving a complete response in multiple myeloma, and the impact of novel agents. J Clin Oncol, 28, 2612-24.

Durie B G, Harousseau J L, Miguel J S, et al (2006). International uniform response criteria for multiple myeloma. Leukemia, 20, 1467-73.

Engelhardt M, Kleber M, Udi J, et al (2010). Consensus statement from European experts on the diagnosis, management, and treatment of multiple myeloma: from standard therapy to novel approaches. Leuk Lymphoma, 51, 1424-43.

Harousseau J L, Attal M, and Avet-Loiseau H (2009). The role of complete response in multiple myeloma. Blood, 114, 3139-46.

Kapoor P, Kumar S K, Dispenzieri A, et al (2013). Importance of achieving stringent complete response after autologous stem-cell transplantation in multiple myeloma.J Clin Oncol, 31, 4529-35. 
Knopf KB, Duh MS, Lafeuille MH, et al (2014). Meta-analysis of the efficacy and safety of bortezomib re-treatment in patients with multiple myeloma. Clin Lymphoma Myeloma Leuk, 14, 380-8.

Koh Y, Lee S Y, Kim I, et al (2014). Bortezomib-associated peripheral neuropathy requiring medical treatment is decreased by administering the medication by subcutaneous injection in Korean multiple myeloma patients. Cancer Chemother Pharmacol, 74, 653-7.

Kumar SK, Rajkumar SV, Dispenzieri A, et al (2008). Improved survival in multiple myeloma and the impact of novel therapies. Blood, 111, 2516-20.

Lu J, Lu J, Chen W, et al (2014). Clinical features and treatment outcome in newly diagnosed Chinese patients with multiple myeloma: results of a multicenter analysis. Blood Cancer $J, 4,239$.

Mateos M V (2010). Management of treatment-related adverse events in patients with multiple myeloma. Cancer Treat Rev, 36, 24-32.

Moreau P, Pylypenko H, Grosicki S, et al (2011). Subcutaneous versus intravenous administration of bortezomib in patients with relapsed multiple myeloma: a randomised, phase 3, non-inferiority study. Lancet Oncol, 12, 431-40.

Morgan GJ (2013). Transplants for the elderly in myeloma. Blood, 122, 1332-4.

Nooka AK, Kaufman JL, Behera M, et al (2013). Bortezomibcontaining induction regimens in transplant-eligible myeloma patients: a meta-analysis of phase 3 randomized clinical trials. Cancer, 119, 4119-28.

Raab MS, Podar K, Breitkreutz I, Richardson PG and Anderson K C (2009). Multiple myeloma. Lancet, 374, 324-39.

Reeder CB, Reece DE, Kukreti V, et al (2010). Once- versus twice-weekly bortezomib induction therapy with CyBorD in newly diagnosed multiple myeloma. Blood, 115, 3416-7.

Richardson PG, Briemberg H, Jagannath S, et al (2006). Frequency, characteristics, and reversibility of peripheral neuropathy during treatment of advanced multiple myeloma with bortezomib. J Clin Oncol, 24, 3113-20.

Richardson PG, Delforge M, Beksac M, et al (2012). Management of treatment-emergent peripheral neuropathy in multiple myeloma. Leukemia, 26, 595-608.

Roschewski M, Korde N, Wu SP and Landgren O (2013). Pursuing the curative blueprint for early myeloma. Blood, 122, 486-90.

Sonneveld P, Goldschmidt H, Rosinol L, et al (2013). Bortezomib-based versus nonbortezomib-based induction treatment before autologous stem-cell transplantation in patients with previously untreated multiple myeloma: a meta-analysis of phase III randomized, controlled trials. $J$ Clin Oncol, 31, 3279-87.

Sonneveld P, Schmidt-Wolf I G, van der Holt B, et al (2012). Bortezomib induction and maintenance treatment in patients with newly diagnosed multiple myeloma: results of the randomized phase III HOVON-65/ GMMG-HD4 trial. $J$ Clin Oncol, 30, 2946-55.

Voorhees P M, Laubach J, Anderson K C and Richardson P G (2013). Peripheral neuropathy in multiple myeloma patients receiving lenalidomide, bortezomib, and dexamethasone (RVD) therapy. Blood, 121, 858.

Wang A, Duan Q, Liu X, et al (2012). (Bortezomib plus lenalidomide/thalidomide)- $v s$. (bortezomib or lenalidomide/ thalidomide)-containing regimens as induction therapy in newly diagnosed multiple myeloma: a meta-analysis of randomized controlled trials. Ann Hematol, 91, 1779-84. 